Z Herz-Thorax- Gefäßchir 2011 · 25:185-186

DOI 10.1007/s00398-011-0863-9

Online publiziert: 13. August 2011

(c) Springer-Verlag 2011
Friedrich-Wilhelm Mohr • Anne-Kathrin Funkat

Klinik für Herzchirurgie, Herzzentrum Leipzig GmbH

\title{
Das Deutsche Aortenklappenregister
} Dokumentation des Fortschritts

Liebe Leserinnen und Leser,

die Behandlungsmethoden kardiovaskulärer Erkrankungen sind in den letzten Jahren einem dramatischen Wandel unterworfen. Dem Ziel folgend, durch weniger invasive Techniken eine erfolgreiche Therapie von KHK oder Klappenerkrankungen zu erreichen, ergab sich eine zu Teilen unkontrollierte Wettbewerbssituation sowohl innerhalb der Herzchirurgie als auch der Kardiologie, ohne dass eine adäquate Qualitätssicherung stattgefunden hätte. Die katheterbasierten Aortenklappenimplantationen (TAKI) stellen die vielleicht revolutionärste Neuentwicklung dar, die den konventionellen Aortenklappenersatz als bisher erfolgreichste herzchirurgische Operation mit langfristig gesicherten Ergebnissen herausfordert. Neben der ohne Zweifel weniger invasiven Implantation selbst sind natürlich die Langlebigkeit der unterschiedlichen neuen, stentbasierten Klappentypen und deren hämodynamische Funktion im Vergleich zur konventionellen Technik zu prüfen. Die frühe Einführung einer DRG für die TAKI-Techniken hat in Deutschland zu einem explosionsartig angestiegenen Einsatz dieser Methoden geführt, der nicht zuletzt durch ausgesprochen professionelles Marketing und Proctoring der involvierten Medizinindustrie maximale Unterstützung fand.

In beiden Fachgesellschaften (DGTHG und DGK) ist erstmalig umfassendes Einvernehmen darüber erzielt worden, dass in diesem Falle eine prospektive Qualitätssicherung mittels einer gemeinsamen Registerstudie nicht nur eine wesentliche Aufgabe, sondern auch eine einmali- ge Chance darstellt. Dies führte zur Gründung des Deutschen Aortenklappenregisters. Der Auftrag zur Durchführung dieser Registerstudie wurde an das BQS-Institut übertragen. Deren finanzielle Absicherung konnte bisher lediglich durch die beteiligten Klappenhersteller erreicht werden; ein entsprechendes Engagement der Krankenkassen gelang jedoch bisher nicht.

\section{( Die Registerstudie ist eine wichtige Aufgabe und eine einmalige Chance zugleich}

Im Deutschen Aortenklappenregister sollen prospektiv risikoadjustierte Daten und längerfristige Ergebnisse aller in Deutschland durchgeführten Aortenklappeneingriffe erhoben und in einem 5-Jahres-Follow-up nachverfolgt werden. Prospektiv ist eine Gesamtpopulation von 80100.00o Patienten mit Aortenklappeneingriffen inklusive 35-40.000 TAKI zu erwarten.

Die aktuelle, gesetzlich geregelte Qualitätssicherung durch das AQUA-Institut erfasst bei weitem nicht alle erforderlichen Daten, um beispielsweise Aussagen zur langfristigen Verbesserung der Lebensqualität nach dem Eingriff treffen zu können. Ein Abgleich mit diesen Daten ist erstrebenswert, um auch die Akutergebnisse derjenigen Klinken zu erfassen, die sich nicht am Register beteiligen.

Vielen Klinikern war bisher nicht bewusst, dass eine gesetzliche Verpflichtung zur Qualitätssicherung nach Aortenklappeninterventionen besteht, welche sich jedoch ausschließlich auf die Hospitalsterb- lichkeit bezieht. Dieses Defizit beseitigt nun das Deutsche Aortenklappenregister, wodurch die Nachhaltigkeit aller Maßnahmen nachvollziehbar wird.

In letzter Zeit sahen sich einige Krankenhausgeschäftsführer bei Budgetverhandlungen seitens der Krankenkassen mit dem Versuch konfrontiert, die Teilnahme am Aortenklappenregister mit der Gestaltung des DRG-Mengengerüsts zu verknüpfen. Einige Geschäftsführer waren ratlos, fragten bei ihrem leitenden Herzchirurgen/Medizincontroller oder Kardiologen/Qualitätsmanager nach und erhielten verschiedene Auskünfte:

1. Die Teilnahme ist nicht verbindlich, die gesetzliche Qualitätssicherung ist ausreichend.

2. Sagen Sie mir warum und wir machen mit.

3. Das Aortenklappenregister läuft sehr erfolgreich, seitdem Sie für die nötigen Dokumentationsarbeiten einen Mitarbeiter in Vollbeschäftigung bezahlen.

Die dritte Antwort ist der anzustrebende Idealzustand, der jedoch tatsächlich bereits an einigen, wenn auch bisher wenigen Kliniken existiert. Die ersten beiden Stellungnahmen sollten deshalb keine Option sein. Dennoch steht das Aortenklappenregister innerhalb der kontrovers diskutierten Themen in vorderster Reihe, denn es werden heiße Eisen angefasst: Die Etablierung der TAKI ist einerseits nicht nur mit hohen Komplikations- und Mortalitätszahlen verbunden, es existiert andererseits angesichts der erzielbaren Erlöse auch ein hoher finanzieller Anreiz. Um 
es ausdrücklich zu formulieren: Aus unserer Sicht ist die kathetergestützte Aortenklappenimplantation ein elegantes neues Verfahren mit dessen Hilfe schon viele Patienten eine erfolgreiche Behandlung inklusive nachfolgender hoher Lebensqualität erfahren haben. Diese technologisch-medizinische Innovation zeigt für viele Hochrisikopatienten eine wirksame Behandlungsperspektive auf. In der anderen Waagschale liegen komplikationsreiche, lange Krankenhausaufenthalte und letztendlich eine hohe Letalität. Was wir also immer noch nicht belegen können: Welche Patienten profitieren besonders von der neuen Technologie? Herr Walther wies in seinem Beitrag in Heft 2 der Zeitschrift für Herz-, Thorax- und Gefäßchirurgie bereits darauf hin: Es fehlt eine randomisierte All-comers-Studie, die jedoch derzeit schwer durchführbar ist, auch weil bei den infrage kommenden Patienten nur eine geringe Compliance zu erwarten ist. Mit Hilfe des Registers wird es möglich sein, einer Antwort näher zu kommen.

Um noch einmal auf die erzielbaren Erlöse zurückzukommen: Für einen konventionellen Aortenklappenersatz betragen die Vergütung näherungsweise 15.00o Euro und die Implantatkosten etwa 1500-2500 Euro. Für eine kathetergestützte Implantation belaufen sich die Kosten auf geschätzte 34.00o Euro als Vergütung bzw. 15.00o Euro für die Klappenprothese. Einkalkuliert werden muss zudem, dass ein Krankenhaus, richtet es sich nach den Empfehlungen des Konsensuspapiers von DGTHG und DGK, für die Einführung der kathetergestützten Technik daneben hohe Investitionskosten $\mathrm{zu}$ schultern hat: die Einrichtung eines Hybrid-Operationssaals und die Schulung der Mitarbeiter. Nicht alle Häuser halten sich jedoch an diese Vorgaben und gefährden damit einen positiven Trend.

Wie ist nun der aktuelle Stand hinsichtlich des Registers?

Derzeit nehmen 80 Krankenhäuser teil, nahezu alle herzchirurgischen Zentren haben inzwischen einen entsprechenden Vertrag mit der BQS abgeschlossen. Es fehlen sicher noch einige Kliniken, in denen ohne eine direkte Beteiligung der Herzchirurgie transfemorale TAKI erfolgen. Hierzu wird ein Abgleich mit dem AQUA-Institut Aufklärung bringen.
Seit Beginn des Registers im Juli 2010 mit zunächst zögerlichem Start konnte ab Januar 2011 eine sehr erfreulich hohe Beteiligung der Zentren beobachtet werden: bis zum 15. Juni 2011 gaben mehr als 9000 Patienten ihre Einwilligung zur Beteiligung an dieser Registerstudie.

Diese gemeinsame erste große Registerstudie von DGTHG und DGK ist ein zukunftsträchtiges Projekt, das sich auch dadurch auszeichnet, dass die Datenerhebung durch eine unabhängige Institution erfolgt. Ziele und Ergebnisse sind in unser aller Interesse und werden auch durch die Deutsche Herzstiftung im Sinne unserer Patienten unterstützt.

In diesem Sinne herzliche Grüße Ihre

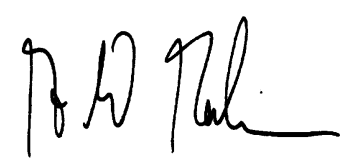

Prof. Dr. Friedrich-Wilhelm Mohr

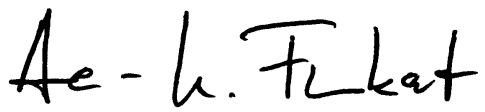

Dr.-Ing. Anne-Kathrin Funkat

\section{Korrespondenzadressen \\ Prof. Dr. Friedrich-Wilhelm Mohr}

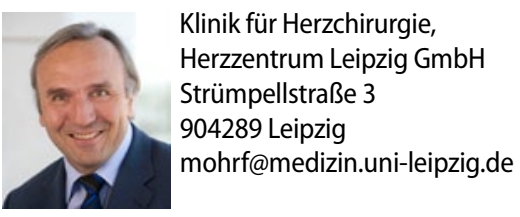

\section{Dr.-Ing. Anne-Kathrin Funkat}

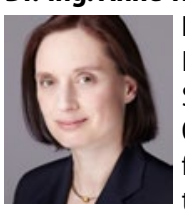

Klinik für Herzchirurgie,

Herzzentrum Leipzig $\mathrm{GmbH}$

Strümpellstraße 39

04289 Leipzig

funkat.ch@herzzen-

trum-leipzig.de

\section{Herz und Hirn}

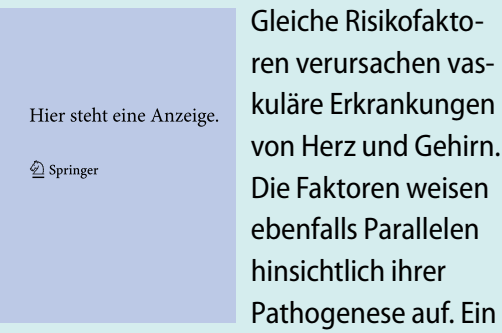

Beispiel ist die zerebrale Ischämie, die mit einem hohen Herzinfarktrisiko einhergeht. Umgekehrt haben Patienten mit koronarer Herzkrankheit und Patienten mit Herzinsuffizienz ein erhöhtes Schlaganfallrisiko. Neue Entwicklungen hinsichtlich der Antikoagulation, der antiarrhythmischen Therapie sowie interventioneller Techniken erweitern das therapeutische Spektrum von Kardiologen und Neurologen.

Das Leitthemenheft "Herz und Hirn" (Ausgabe 02/11) der Zeitschrift Der Nervenarzt informiert über Innovationen auf diesem

Themengebiet. Es beinhaltet u.a. Beiträge zu folgenden Themen:

- Entwicklung von Mortalität und Morbidität vaskulärer Erkrankungen

- Medikamentenadhärenz bei chronischen Erkrankungen

- Epidemiologie, Klinik und Management des Vorhofflimmerns

- Detektion von Vorhofflimmern beim Schlaganfall

- Techniken zum Verschluss des linken Herzohres

- Neue orale Antikoagulanzien beim Vorhofflimmern

- Kognitive Störungen nach kardiochirurgischen Eingriffen

Bestellen Sie diese Ausgabe zum Preis von EUR 34,-zzgl. Versandkosten bei:

Springer Customer Service Center Kundenservice Zeitschriften

Haberstr. 7, 69126 Heidelberg

Tel.: +49 6221-345-4303

Fax: +49 6221-345-4229

E-Mail: leserservice@springer.com

P.S. Vieles mehr rund um Ihr Fachgebiet finden Sie auf www.springermedizin.de 